\title{
DYNAMIC MODULI AND LOCALIZED DAMAGE IN COMPOSITES
}

\author{
L.S. Fu \\ The Ohio State University \\ Columbus, Ohio 43210
}

The alm in nondestructive evaluation (NDE) by sound and ultrasound is to extract material and fracture properties from pulses sent by and recelved at transducers. The analysis is usually given either in the time domain or the frequency domain. The introduction of the size factor, the wavelength, in the model mechanics problems in NDE plays the important role of relating the far-field measurable quantities such as phase velocities and attenuation to the near-field physical situation such as local geometric dimensions and elastic properties. This aspect of the analysis thus allows "nondestructive" testing methods to be employed for "experimental" validation of the predictions by the theories (refs. 1 to 3 ).

Solution to the elastic wave scattering due to a single embedded inhomogeneity is available by different methods that are appropriate at different frequency ranges. The methods that offer a solution in an analytic form and are useful for inhomogeneous media with multiple components are the long-wave approximation, the polarization approach, and the extended method of equivalent inclusion (refs. 4 and 5 ).

Several averaging schemes or theorems exist in the literature for finding the dynamic effective moduli and mass density. Some efforts concentrate on the average stress $\mathscr{\sigma}$ and strain $\varepsilon$ fields or on the average displacement field $\underset{\sim}{u} ;$ others use a variational approach. These theories are appropriate mostly at Rayleigh or longwave limits and do not exhibit dispersive effects. Dispersiveness and attenuation are important in evaluating dynamic material properties.

This study dealt first with the scatter of elastic waves due to a thin, flat ellipsoidal inhomogeneity, either penny shaped or elliptical. An average theorem appropriate for dynamic effective mass density and effective moduli was developed via a self-consistent scheme. Effective material properties of two-component media consisting of randomly distributed spheres are given here as a special case.

\section{PRELIMINARIES}

Displacement Field due to Presence of Mismatch

The inhomogeneous media considered in this paper are assumed to consist of a homogeneous matrix of elastic moduli $C^{0}$ and mass density $\rho^{0}$ and a distribu. tion of inhomogeneities with moduli $\underset{\sim}{\sim}(r)$ and mass density ${ }_{p}(r)$ occupying regions $\Omega_{r}, r=1,2, \ldots, n$ (fig. 1). The total displacement field $\underline{u}$ can be separated into two parts.

$$
\underline{u}=\underline{u}^{(i)}+\underline{u}^{(m)}
$$

where the superscripts ( $i$ ) and ( $m$ ) denote "incident" and "mismatch, "respectively. It is clear that when no mismatch components (i.e., inhomogeneities) are present, 
the total displacement field is entirely the same as the incident displacement wave field. On the other hand, if there is no incident wave field, the only field that exists is the null field.

\section{Eigenstrains}

The eigenstrains $\varepsilon^{*}$, which are also termed transformation strains, are defined as the part of the total strain $\varepsilon$ that must be subtracted before the remaining part can be related to stresses $\underset{\sim}{\sim}$ through Hooke's law

$$
\begin{gathered}
\varepsilon_{r s}^{*}=\varepsilon_{r s}-\varepsilon_{r s}^{e} \\
\varepsilon_{r s}^{e}=c_{j k r s}^{-1} \sigma_{j k}
\end{gathered}
$$

where $\mathcal{C}^{-1}$ are the elastic compliances. The method of equivalent inclusion is a method that allows the inhomogeneous media to be replaced by media of homogeneous matrix effective moduli with distributed transformation strains in the regions originally occupied by inhomogeneities; hence

$$
\varepsilon_{r s}^{*}= \begin{cases}0 & \text { in } \Omega_{0} \\ \varepsilon_{r s}^{*} & \text { in } \Omega_{r}, r=1,2, \ldots, n\end{cases}
$$

For the two problems to be equivalent, the transformation strains must give rise to a field that is exactly the same as the "mismatch" field $\underset{\sim}{\mathrm{u}}(\mathrm{m})$. This leads to equivalence conditions that ensure that identical field quantities at any given point will be obtained in the two problemst (ref. 4):

$$
\begin{aligned}
& \Delta C_{j k r s} u_{r, s}^{(m)}(\bar{r})+C_{j k r s} \varepsilon_{r s}^{*}(1)(\bar{r})=-\Delta C_{j k r s} u_{r, s}^{(1)}(\bar{r}) \quad \text { in } \Omega \\
& \Delta \rho \omega^{2} u_{j}^{(m)}(\bar{r})+c_{j k r s} \varepsilon_{r s, k}^{*(2)}(\bar{r})=-\Delta \rho \omega^{2} u_{j}^{(1)}(\bar{r}) \quad \text { in } \Omega
\end{aligned}
$$

Two types of transformation strains, or eigenstrains, arise in elastodynamic situations due to the mismatch in elastic moduli $\Delta c$ and mass density $\Delta \rho$. It is often convenient and useful to define associated quantities such as

$$
\begin{gathered}
m_{j k}=c_{j k r s}{ }^{*}{ }_{r s}(1) \\
\pi_{j}=c_{j k r s^{\varepsilon} r s, k}{ }^{*(2)}
\end{gathered}
$$

where $m_{j k}$ and $\pi_{j}$ are moment density tensor and equivalent force or eigenforce, respectively.

the conditions (eqs. (5) and (6)) are similar to those of willis (1980) and those of Mura, Proc. Int. Conf. on Mechanical Behavior of Materials, $\underline{5}$, Socjety of Materials Science, Japan, 12-18 (1972). 
The volume average and time average of a field quantity, say $F(r, t)$, are denoted by using brackets \langle\rangle$_{V}$ and \langle\rangle$_{T}$, respectively, and are defined as

$$
\begin{aligned}
& \langle F(\underline{r}, t)\rangle=\frac{1}{V} \int F(\underline{v}, t) d V \\
& \langle F(\underline{r}, t)\rangle=\frac{1}{T} \int F(\underline{r}, t) d T
\end{aligned}
$$

where $V$ and $T$ are volume and time period, respectively, and $\underline{v}$ is velocity field.

\section{Volume Integrals of Ellipsoid Associated with Inhomogeneous Helmholtz Equation}

Volume integrals of an ellipsoid associated with the integration of the inhomogeneous Helmholtz equation are used in this work. The inhomogeneous scalar Helmholtz equation takes the form

$$
\nabla^{2} \Phi+k^{2} \Phi=-4 \pi \gamma(\underline{r})
$$

where $r(\underline{r})$ is the source distribution or density function and $\nabla^{2}$ and $k$ are the Laplacian and wave number, respectively. A particular solution to equation (11) is

$$
\Phi(\underline{r})=\int_{\Omega} Y\left(\underline{r}^{\prime}\right) R^{-1} \exp (i k R) d V^{\prime}, R=\left|\underline{r}-\underline{r}^{\prime}\right|
$$

in which $(4 \pi R)^{-1} \exp (i k R)$ is the steady-state scalar wave Green's function and $\Omega$ is the region where the source is distributed. The source distribution function $r(\underline{r})$ can be expanded in basic functions or polynomial form, depending on the geometry of the volumetric region $\Omega$. For an ellipsoidal region, the choice of using a polynomial expansion separates this work from other theories of elastic wave scattering:

$$
\gamma\left(\underline{r}^{\prime}\right)=\left(x^{\prime}\right)^{\lambda}\left(y^{\prime}\right)^{\mu}\left(z^{\prime}\right)^{v}
$$

in which $\lambda, \mu$, and $v$ are integers and $k$ is either longitudinal or transverse wave number.

For elastic wave scattering in an isotropic elastic matrix, two types of volume integrals and their derivatives must be evaluated: The $\psi$-integrals are given by

$$
\begin{aligned}
\psi(\underline{r}) & =\int_{\Omega} R^{-1} \exp (i \alpha R) d V^{\prime} \\
\psi_{k}(\underline{r}) & =\int_{\Omega} x_{k}^{\prime} R^{-1} \exp (i \alpha R) d V^{\prime} \\
\psi_{k \ell \ldots s}(\underline{r}) & =\int_{\Omega} x_{k}^{\prime} x_{\ell}^{\prime} \ldots x_{s}^{\prime} R^{-1} \exp (i \alpha R) d V^{\prime}
\end{aligned}
$$




$$
\begin{aligned}
& \psi,_{p}(\underline{r})=\frac{\partial}{\partial x_{p}} \psi(\underline{r}) \\
& \psi_{k, p}(\underline{r})=\frac{\partial}{\partial x_{p}} \psi_{k}(\underline{r})
\end{aligned}
$$

where $\alpha^{2}=\omega^{2} / V^{2}=\rho \omega^{2} /(\lambda+2 \mu)$. The other type, the $\phi$-integrals, are obtained by replacing $\alpha$ with $\beta$ in equations (12) and (13), where $\beta^{2}=\rho \omega^{2} /(\mu)$. Details of the integration are given in reference 6 . In this work, only the limiting values at $r \rightarrow 0$ and $r \rightarrow \infty$ are of interest.

\section{ISOLATED, FLAT ELLIPSOIDAL INCLUSION}

Formulation

Consider the physical problem of an isolated inhomogeneity embedded in an infinite elastic solid that is subjected to a plane time-harmonic incident wave field as depicted in figure 1. Replacing the inhomogeneity with the same material as that of the surrounding medium, with moduli $c_{j k r s}$ and mass density $\rho$, and including in this region a distribution of eigenstrains and eigenforces, the physical problem is now replaced by the equivalent inclusion problem.

The total field is now obtained as the superposition of the incident field and the fleld induced by the presence of the mismatches in moduli and in mass density written in terms of eigenstrains $\varepsilon_{i j}^{*}(1)$ and eigenforces $\pi_{j}^{*}$

$$
\underset{\sim}{F}={\underset{\sim}{F}}^{(i)}+\underset{\sim}{F}(m)
$$

where $\underset{F}{ }$ denotes either the displacement field $u_{j}$, the strain field $\varepsilon_{i j}$, or the stress field $\sigma_{i j}$.

For uniform distributions of eigenstrains and eigenforces, the fields can be obtained as

$$
\begin{gathered}
u_{m}^{(m)}\left(\underline{r}^{\prime}\right)=-\pi_{j}^{*} S_{j m^{\prime}}\left(\underline{r}^{\prime}\right)-c_{j k r s^{\varepsilon}}^{*}(1) S_{j m, k^{\prime}}\left(\underline{r}^{\prime}\right) \\
\varepsilon_{m n}^{(m)}=\left[u_{m, n}^{(m)}+u_{n, m}^{(m)}\right] / 2 \\
\sigma_{p q}^{(m)}=c_{p q m n} \varepsilon_{m n}^{(m)}
\end{gathered}
$$

where a comma denotes partial differentiation and

$$
S_{j m}(\underline{r})=\int_{\Omega} g_{j m}\left(\underline{r}-\underline{r}^{\prime}\right) d V^{\prime}
$$

in which $g_{j m}$ is the spatial part of the steady-state elastic wave Green's function and $\Omega$ is the region occupied by the inhomogeneity. The integrals $S_{j m}$ and their derivatives must be evaluated for the regions $\underline{r}>\underline{r}^{\prime}$ and $\underline{r}<\underline{r}^{\prime}$ 
(ref. 6). The solution form represented in equations (14) to (18) gives the fields inside and outside an isolated inhomogeneity of arbitrary shape.

\section{Far-Field Scattered Quantities}

Let the incident displacement fleld be longitudinal and of frequency $\omega$ and amplitude $u_{0}$ :

$$
u_{j}^{(1)}=u_{0} q_{j} \exp \left(i \alpha x_{1} k_{1}-i \omega t\right)
$$

where $i^{2}=-1$ and $q_{j}$ is the unit vector in the normal direction of the plane time-harmonic incident wave and $k_{j}$ is wave vector. For a linear isotropic medium, the spatial part of the free-space Green's function is well known. Substituting $g_{j m}\left(r-r^{\prime}\right)$ in equations (18) and (20) and using the limiting concept

$$
\begin{aligned}
& \lim _{a_{3 \rightarrow 0}} a_{3} \pi_{j}^{*}=A_{j} \quad \text { constants } \\
& \lim _{a_{3} \rightarrow 0} a_{3} \varepsilon_{i j}^{*}(1)=B_{i j} \text { constants }
\end{aligned}
$$

the scattered displacement $u_{m}(s)(r, t)$ from a thin, elliptical flat crack can easily be obtained as

$$
\begin{aligned}
\frac{u_{m}^{(s)}(\underline{r}, t)}{\left(\alpha a_{1}\right)^{3} u_{0}} & =\left.\frac{u_{m}^{(m)}(\underline{r}, t)}{\left(\alpha a_{1}\right)^{3} u_{0}}\right|_{r \rightarrow \infty} \\
& =\left[\left(C G_{m} \exp i \alpha r\right) / \alpha r+\left(D H_{m} \exp i \beta r\right)\right] \beta r \quad \exp (-i \omega t)
\end{aligned}
$$

where

$$
\begin{gathered}
G_{m}=-\left(a_{2} / a_{1}\right)\left[-l_{m} \ell_{j} A_{j}^{*}+\left(1-2 \alpha^{2} / \beta^{2}\right) l_{m} B_{j j}^{*}+2\left(\alpha^{2} / \beta^{2}\right) l_{m} l_{k} \ell_{j} B_{k j}^{*}\right] \\
H_{m}=\left(a_{2} / a_{1}\right)\left[-(\beta / \alpha)^{3}\left(l_{m} \ell_{j}-\delta_{m j}\right) A_{j}^{*}-2(\beta / \alpha)^{2} \ell_{k} B_{k m}^{*}+2(\beta / \alpha)^{2} \ell_{m} \ell_{k} \ell_{j} B_{k j}^{*}\right] \\
C=\left[j_{0}\left(\alpha r_{e}\right)+j_{2}\left(\alpha r_{e}\right)\right] / 3=\sin \alpha r_{e} /\left(\alpha r_{e}\right)^{3}-\cos \alpha r_{e} /\left(\alpha r_{e}\right)^{2} \\
D=\left[j_{0}\left(\beta r_{e}\right)+j_{2}\left(\beta r_{e}\right)\right] / 3=\sin \beta r_{e} /\left(\beta r_{e}\right)^{3}-\cos \beta r_{e} /\left(\beta r_{e}\right)^{2} \\
r_{e}^{2}=a_{i}^{2} l_{j}^{2}, \quad a_{3}=0
\end{gathered}
$$

in which $m, j, k=1,2,3$ and $\left(a_{1}, a_{2}\right), \ell_{m}, \alpha$, and $\beta$ denote the semiaxes of the flat ellipsoid, direction cosines of scattered displacements, longitudinal wave 
number, and shear wave number, respectively. Also, $A_{j}^{\star}$ and $B_{j k}^{*}$ are the reduced nondimensional forms of $A_{j}$ and $B_{j k}$, respectively, defined as follaws:

$$
\begin{gathered}
A_{j}^{*}=A_{j} /\left(\Delta \rho \omega^{2} u_{0}\right), \quad \Delta \rho=\rho^{\prime}-\rho \\
B_{j k}^{*}=-B_{j k} /\left(i \alpha u_{0}\right)
\end{gathered}
$$

Expressions for the differential cross section $\mathrm{dP}(\omega) / \mathrm{d} \Omega$ and the total cross section $P(\omega)$ can be obtained as (ref. 5):

$$
\begin{gathered}
\frac{d P(\omega)}{d \Omega}=\sigma^{L}(\theta, \phi)+(\alpha / \beta) \sigma^{\top}(\theta, \phi) \\
P(\omega)=\int\left[\sigma^{L}(\theta, \phi)+(\alpha / \beta) \sigma^{\top}(\theta, \phi)\right] d \Omega
\end{gathered}
$$

where $d \Omega$ is the differential element of solid angle and

$$
\begin{aligned}
& \alpha^{2} \sigma^{L}(\theta, \phi)=\left(\alpha a_{1}\right)^{6}\left[\mathrm{CG}_{m}\right]\left[\overline{\mathrm{CG}_{m}}\right] \\
& \beta^{2} \sigma^{T}(\theta, \phi)=\left(\alpha a_{1}\right)^{6}\left[D H_{m}\right]\left[\overline{D H_{m}}\right]
\end{aligned}
$$

in which the overbars denote complex conjugates. The constants $A_{j}^{*}$ and $B_{j k}^{*}$ must be evaluated from the equivalence conditions (eqs. (5) and (6)) with the use of the limiting concepts in equations (21) and (22) and of the integration method developed in reference 6 .

\section{Determination of $A_{j}^{*}$ and $B_{j k}^{*}$}

In equations (21) to (25) the scattered displacement field is given in terms of the "reduced' forms of the eigenforces and eigenstrains (1.e., $A_{j}^{*}$ and $B_{j k}^{*}$ ). These constants must in turn be determined from the equivalence conditions. By writing the incident wave field in a Taylor series, the governing simultaneous algebraic equations can be easily obtained. Since $f_{i j}[0]$ and $F_{m i j}[0]$ vanish automatically, these governing equations become uncoupled and lead to a three-bythree system for $A_{j}^{*}$ and a six-by-six system for $B_{j k}^{*}$. For a linear elastic medium, they are

$$
\Delta \rho \omega^{2} u_{0} f_{j s}[0] A_{j}^{*}+A_{s}^{*}=-q_{s}
$$

$\left\{\Delta \lambda \delta_{s t} D_{m m j j}[0]+2 \Delta \mu D_{s t j k}[0]\right\} B_{j k}^{*}+\left(\lambda \delta_{s t} B_{m m}^{*}+2 \mu B_{s t}^{*}\right)$

$$
=-\left(\Delta \lambda \delta_{s} q_{m} q_{m}+2 \Delta \mu q_{s} q_{t}\right)
$$

where the subscripts $s, t, m, j, k=1,2,3$ denote sum from 1 to 3 and

$$
4 \pi \rho \omega^{2} f_{j s}(\underline{r})=-\beta^{2} \phi \delta j s+\psi, m j-\phi, m j
$$


$4 \pi \rho \omega^{2} D_{s t j k}(r)=2 \mu(\psi, s t j k+\phi, s t j k)-\mu \beta^{2}(\phi, j t \delta k s+\phi, j s \delta k t)-\lambda \alpha^{2} \psi, m n \delta_{j k}$ in which

$$
\Delta \lambda=-\lambda, \quad \Delta \mu=-\mu, \quad \Delta \rho=-\rho
$$

The $\phi$ - and $\psi$-integrals and their derivatives are evaluated by the method suggested in reference 6 . Retaining terms up to $\alpha_{1}$ or $\mathrm{Ba}_{1}$ of the fourth order, the constants are obtained as

$$
\begin{gathered}
A_{j}^{*}=-q_{j} /\left\{u_{0} \omega^{2} \Delta \rho f_{j}[0]+1\right\}, \text { no sum on } j \\
f_{j}[0]=\left(f_{11}[0], f_{22}[0], f_{33}[0]\right) \\
\left\{B_{i}^{*}\right\}=\left[b_{i j}\right]^{-1}\left[c_{j}\right], 1, j=1,2,3
\end{gathered}
$$

$\left\{B_{i j}^{*}\right\}=-q_{i} q_{j} /\left[1+\xi\left(\phi_{j}[0]+\phi_{j}[0]\right)\right]$, no sum on $i, j$

$$
i \neq j ; i, j=i, 2,3 ; \xi=1 / 4 \pi
$$

where in equation (37) $B_{1}^{*}=B_{11}^{*}, B_{2}^{*}=B_{22}^{*}, B_{3}^{*}=B_{33}^{*}$, and

$$
\begin{gathered}
c_{j}=(\Delta \lambda+2 \Delta \mu)\left(1+q_{j}^{2}\right), j=1,2,3 \\
b_{11}=(\lambda+2 \mu)+\lambda \zeta \psi, j j[0]+2(\lambda+2 \mu) \xi \phi, 11[0]+2 \mu \zeta \psi, 11[0] \\
b_{12}=\lambda+\lambda \zeta \psi, j j[0]+2 \mu \zeta \psi,{ }_{11}[0]+2 \xi \lambda \phi, 22[0] \\
b_{13}=\lambda+\lambda \zeta \psi, j j[0]+2 \mu \zeta \psi,{ }_{11}[0]+2 \lambda \xi \phi,{ }_{33}[0] \\
b_{21}=\lambda+\lambda \zeta \psi, j j[0]+2 \mu \zeta \psi, 22[0]+2 \xi \lambda \phi,{ }_{11}[0] \\
b_{22}=(\lambda+2 \mu)+\lambda \zeta \psi, j j[0]+2 \mu \zeta \psi, 22[0]+2 \xi(\lambda+2 \mu) \phi, 22[0] \\
b_{23}=\lambda+\lambda \zeta \psi, j j[0]+2 \mu \zeta \psi, 22[0]+2 \xi \lambda \phi,{ }_{33}[0] \\
b_{31}=\lambda+\lambda \zeta \psi, j j[0]+2 \mu \zeta \psi, 33[0]+2 \lambda \xi \phi,{ }_{11}[0] \\
b_{32}=\lambda+\lambda \zeta \psi, j j[0]+2 \mu \zeta \psi,{ }_{33}[0]+2 \lambda \xi \phi,{ }_{22}[0] \\
b_{33}=(\lambda+2 \mu)+\lambda \zeta \psi, j j[0]+2 \mu \zeta \psi, 33[0]+2 \xi \phi,{ }_{33}(\lambda+2 \mu)[0]
\end{gathered}
$$

Note that $b_{1 j} \neq b_{j 1}$. In equation $(38), \phi_{1}=\phi_{11}[0], \phi_{2}=\phi_{22}[0]$, and $\phi_{3}=\phi_{33}[0]$. The $f-$ and $\phi-$ functions are given as

$$
\begin{gathered}
4 \pi \rho \omega^{2} f_{j s}[0]=-\beta^{2} \phi[0] \delta_{j s}+\psi, j s[0]-\phi, j s[0] \\
\beta_{\phi}^{2}[0]=\pi a_{1} a_{2} \beta^{2}\left\{I_{0}-\left[\left(\beta a_{1}\right)^{2} / 16\right] I_{1}+1(8 / 3) \beta\right\} \\
\phi,{ }_{17}[0]=-\left[\left(\pi a_{1}^{3} a_{2} \beta^{4}\right) / 12\right] I_{1}
\end{gathered}
$$




$$
\begin{gathered}
\phi, 22[0]=-\left[\left(\pi a_{1}^{3} a_{2} \beta^{4}\right) / 12\right] \quad I_{1} \\
\phi \cdot 33[0]=0 .
\end{gathered}
$$

in which

$$
\begin{gathered}
I_{0}=\int_{0}^{\infty} \frac{d \psi}{\Delta(\psi)}=F(\theta, k) \frac{2}{a_{1}} \\
I_{1}=\int_{0}^{\infty} \frac{\psi d \psi}{\left(a_{1}^{2}+\psi\right) \Delta(\psi)}=\frac{2}{a_{1}}\left[\frac{E(\theta, k)}{k^{2}}-\frac{k^{\prime 2}}{k^{2}}\right] F(\theta, k) \\
I_{2}=\int_{0}^{\infty} \frac{\psi d \psi}{\left(a_{2}^{2}+\psi\right) \Delta(\psi)}=\frac{2}{a_{1}}\left[\frac{F(\theta, k)}{k^{2}}-\frac{E(\theta, k)}{k^{2}}\right] \\
F=\int_{0}^{\theta} \frac{d \omega}{\left(1-k^{2} \sin ^{2} \omega\right)^{1 / 2}}, E=\int_{0}^{\theta}\left(1-k^{2} \sin ^{2} \omega\right)^{1 / 2} d \omega
\end{gathered}
$$

and as $a_{3} \rightarrow 0, \theta \rightarrow \pi / 2, k^{2} \rightarrow\left(1-a_{2}^{2} / a_{1}^{2}\right)$ and $k^{\prime 2}=\left(1-k^{2}\right) \rightarrow a_{2}^{2} / a_{1}^{2}$, if $a_{1}>a_{2}$. If $a_{3} \rightarrow 0$ and $a_{1}=a_{2}$, we have $I_{0}=\pi / a_{1}$ and $I_{1}=I_{2}=\pi / 2 a_{1}$. The $\psi$-functions are obtained by replacing $\beta$ with $\alpha$ in the $\phi$-functions (fig. 2).

\section{Numerical Calculations and Graphical Displays}

It is clear from equations (37) to (40) that the uniformly distributed eigenstrains and eigenforces in their reduced forms, $B_{i j}$ and $A_{j}$, respectively, depend only on the characteristics of the incident wave field and the geometric factors of the inhomogeneity for a given material system. It is observed from equations (37) to (41) that $B_{j}$ and $B_{i j}$ as functions of dimensionless wave number $\alpha a_{1}$ or $\alpha a_{2}$ would exhibit large peak values at certain incident wave frequencies when $b_{i j} \rightarrow 0$. The values of these critical frequencies depend only on the matrix elastic moduli, the crack dimensions, and the measurement direction. For a given aspect ratio $a_{2} / a$ and a measurement direction, the difference in frequencies at subsequent peak values is proportional to $a_{7}$, the largest dimension of the inclusion.

Computational data of elastic wave scattering due to a flat embedded inhomogeneity in any given isotropic material system can be obtained by employing equations (24) to (26) and (33) to (50). Scattered displacement amplitudes can easily be obtained for any given aspect ratio. In figures 3 and 4 , computational data for the back-scattered situation are displayed for a tungsten disk in an aluminum matrix for aspect ratios $a_{2} / a_{1}=0.01$ and $a_{2} / a_{1}=0.50$. In figures 5 to 8 , critical frequencies were observed in $B_{i j}^{*}$, but no critical frequencies were identified for $A_{j}^{\star}(j=1,2,3)$. The critical frequencies for $C\left|G_{m}\right|$ and $D\left|H_{m}\right|$ are, however, clearly identified. The position along the aa axis at which the first critical 
frequency occurred depended on the aspect ratio and matrix elastic moduli. A sufficientiy smali increment in al must be used in order not to miss any peak values. Since the solution form given in equations (24) to (26) and (33) to (50) is analytic in frequency, this can easily be achieved. Most of the scattered energy is carried by the transverse components of the scattered displacement ( $1 . e .$, $\left|D H_{m}\right|>\left|C G_{m}\right|$, figs. 3 and 4$)$.

\section{DYNAMIC MODULI AND DAMAGE OF COMPOSITES}

Consider the problem of an inhomogeneous medium as 117ustrated in figure 9 , under a plane time-harmontc incident wave fleld. The true composite thus occupies the whole region and possesses effective moduli $\mathcal{C}^{*}$ and mass density $\rho^{*}$. To determine the effective moduli and mass density, an average strain energy and kinetic energy were used. The effective properties were found to depend on a fourth-rank tensor $\underset{\sim}{A}$ and a second-rank tensor $\underset{\sim}{D}$. A self-consistent scheme was then developed for determining these tensors.

\section{Average Theorem}

To determine the effective moduli and mass density, the following definitions were used:

$$
\begin{gathered}
\langle\underline{q}\rangle=\mathscr{c}^{*}\langle\underline{q}\rangle \\
\langle\underline{q} \underline{\sim}\rangle=\underline{\mathcal{C}}^{*}\langle\underline{\varepsilon}\rangle\langle\underline{\varepsilon}\rangle \\
\langle\rho \underline{v}\rangle=\rho^{*}\langle\underline{v}\rangle \\
\langle\rho \underline{v} \cdot \underline{v}\rangle=\rho^{*}\langle\underline{v}\rangle\langle\underline{v}\rangle
\end{gathered}
$$

where $g, \underset{\sim}{\mathcal{E}} \underline{v}$ are the stress, strain, and velocity fields, the $\sim$ denotes a tensoriai quantity, and the angular brackets $\langle$ denote the volume average of a field quantity (eq. (9)). The left and the right sides of equations (52) and (54) can be shown to be equivalent under the so-called Hill's condition ( $r e f .7),\langle\sigma\rangle\langle\varepsilon\rangle$ $=\langle\sigma \varepsilon\rangle$. From equations (53) and (54), it is clear that the kinetic energy per unit volume of the effective medium can be made equal to that of the physical medium if, and only if, a frequency-dependent mass density is defined. This is the same as requiring, by again using the Hill's conditions $\langle p, \underline{v} \cdot \underline{v}\rangle=\langle p \underline{v}\rangle\langle\underline{v}\rangle$, the average linear momentum per unit volume to be the same as the effective unit volume (eq. (53)). These conditions (eqs. (53) and (54)) are not met if the static definition of effective mass density is used.

Let $f_{r}$ denote the volume fraction of the $r_{\text {th }}$ inclusion material. Then the volume averages of the stress and velocity fields $\sigma$ and $u$, respectively, are

$$
\begin{aligned}
& \langle\underline{\sigma}\rangle=\sum_{r=0}^{n} f_{r{ }^{\sigma}}(r) \\
& \langle\underline{\dot{u}}\rangle=\sum_{r=0}^{n} f_{r} \underline{\dot{u}}^{(r)}
\end{aligned}
$$


where $\underset{\sim}{g}(r)=\underset{\sim}{\stackrel{c}{c}}(r) \underset{\sim}{g}(r),(r)=0,1,2, \ldots, n$ and for a time-harmonic situation

$$
\underline{\dot{u}}=\underline{v}=-i \omega \underline{u}
$$

Equations (52) and (54) can be rearranged, by using equations (55) and (56), as

$$
\begin{gathered}
\langle\underline{q \varepsilon}\rangle=c^{0}\langle\underline{\varepsilon}\rangle\langle\underline{\varepsilon}\rangle+\sum_{r=1}^{n} f_{r} \mathcal{c}^{(r)_{\varepsilon}} \underset{\sim}{(r)}\langle\underline{\varepsilon}\rangle \\
\langle\rho \underline{v} \cdot \underline{v}\rangle=\rho^{0}\langle\underline{v}\rangle\langle\underline{v}\rangle+\sum_{r=1}^{n} f_{r} \Delta \rho(r) \underline{v}^{(r)}\langle\underline{v}\rangle
\end{gathered}
$$

Consider now the case where the solution form possesses a linear relation between the velocity and strain fields in the $r^{\text {th }}$ component and the average velocity and strain fields of the effective medium, that is,

$$
\begin{aligned}
& \underline{\varepsilon}^{(r)}=\stackrel{A}{\sim}^{(r)}\langle\varepsilon\rangle \\
& \underline{v}(r)=\underset{\sim}{\stackrel{D}{\sim}(r)}\langle\underline{v}\rangle
\end{aligned}
$$

where $\underset{\sim}{A}(r)$ and $\underset{\sim}{D}(r)$ are tensors of fourth and second rank that must be

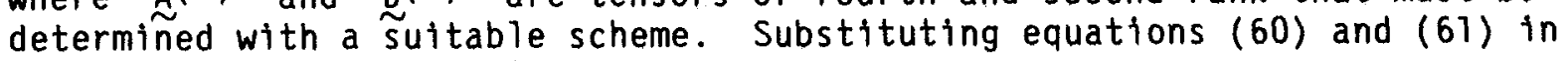
equations (58) and (59) leads to

$$
\begin{aligned}
& \langle\tilde{\sigma} \boldsymbol{\sim}\rangle=\left[\mathcal{L}^{0}+\sum_{r=1}^{n} f_{r} \Delta \mathcal{C}^{(r)_{A}}(r)\right]\langle\underline{\varepsilon}\rangle\langle\varepsilon\rangle \\
& \langle\rho \underline{v v}\rangle=\left[\rho^{0}+\sum_{r=1}^{n} f_{r} \Delta \rho(r)_{D}(r)\right]\langle\underline{v}\rangle\langle\underline{v}\rangle
\end{aligned}
$$

Comparing these equations with equations (52) and (54) gives the following expressions for the dynamic effective moduli and mass density:

$$
\begin{aligned}
& \stackrel{\mathcal{L}}{*}^{*}=\mathcal{L}^{0}+\sum_{r=1}^{n} f_{r} \Delta \mathcal{L}^{(r)} \stackrel{\mathcal{A}}{(r)}^{(r)} \\
& \left.\begin{array}{c}
\rho^{*}=\rho^{0}+\sum_{r=1}^{n} f_{r} \Delta_{\rho}(r)_{0}(r) \\
D^{(r)}=D_{j j}^{(r) / 3}
\end{array}\right\}
\end{aligned}
$$

Note that the tensors $A^{(r)}$ and $D^{(r)}$ are frequency dependent and replace the static expressions when frequency approaches zero and when proper care is taken. The assumption of a general 1inear dependence between $\underline{v}(r)$ and $\langle\underline{v}\rangle$ (eq. (61) must be specialized such that the second-rank tensor $D^{-}$will degenerate into a scalar. This specialization is automatic for randomly distributed spheres where $D_{m j}=D \delta_{m j}$, in which $D=D_{11}=D_{22}=D_{33}$. 
To determine the explicit form of the tensors $\underset{\sim}{A}(r)$ and $\underset{\sim}{\sim}(r)$, the strain and velocity fields in the $r^{\text {th }}$ component are determined by using the method of equivalent inclusion as presented in the previous section.

\section{Self-Consistent Scheme for Determining Effective Properties}

The tensor fields $\underset{\sim}{A}(r)$ and $\underset{\sim}{D}(r)$ are of ranks four and two, respectively, and are functions of wave numbers, geometric properties, and effective and inclusion material properties. Let the average strain and velocity be the same as those derived from the incident wave field. Then the governing conditions for determining $\underset{\sim}{A}(r)$ and $\underset{\sim}{(r)}$ for the $r^{\text {th }}$ inclusion are simply obtained by rewriting the equivalence conditions for an effective medium with mass density $\rho^{*}$ and moduli $C^{*}$ :

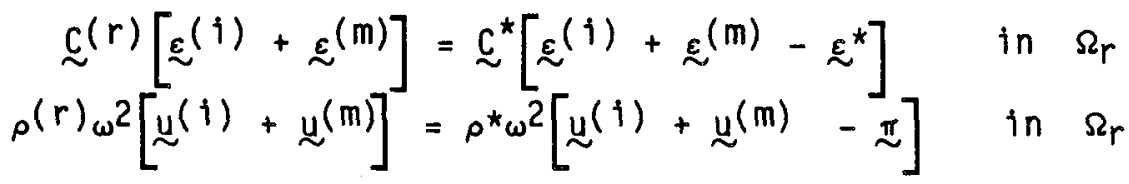

in which the superscripts (m) and (i) denote mismatch and incident, respectively. Slightly different approaches for finding effective moduli for heterogeneous materials that apply to static cases with different constituents and situations are given in references 7 and 8 . The strengths and dynamic responses of composites have been investigated (refs. 9 and 10) and reviewed (refs. 11 and 12).

\section{Effective Properties of Two-Component Media: Randomly Distributed Spheres}

Let the moduli, mass density for the matrix, inclusion material particles, and the effective medium by denoted by $C_{j k r s,}^{0} \rho^{0} ; C_{j k r s}, \rho^{\prime}$; and $C_{j k r s}^{*}$ and $\rho^{*}$, respectively. By using the elastodynamic solution for a single elipsoidal inhomogeneity, the displacement and strain fields inside an inhomogeneity are found, when the average displacements are made equal to the plane time-harmonic incident wave field:

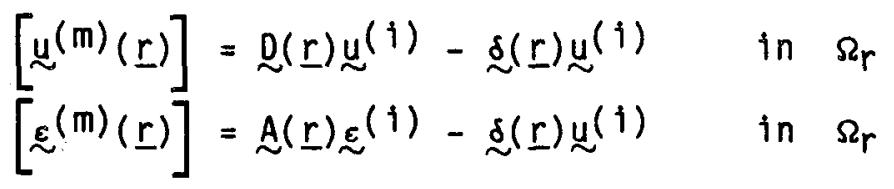

Employing the volume-averaging process as described by equation (9) and substituting in equations (58) and (59), the effective properties are easily defined as follows:

$$
\begin{gathered}
\rho^{*}=\rho+f \Delta \rho D, \quad D=\frac{1}{3} D_{j j} \\
\mathscr{C}^{*}=\underset{\sim}{C}+\stackrel{\Delta C A}{\sim}
\end{gathered}
$$

where $f$ is the volume fraction of inclusion material. The tensor fields $\underset{\sim}{D}$ and $\stackrel{A}{\sim}$ are

$$
D_{m j}=-\left\langle f_{m j}(\underline{r})\right\rangle /\left\{f_{M J}[0]+4 \pi\left(p^{\prime}-p^{\star}\right) \omega^{2}\right\} \text { no sum on } M, J
$$




$$
A_{m n p q}=\left\langle F_{m j k, n}(\underline{r})+F_{n j k, m}(\underline{r})\right\rangle S_{j k p q} / 2 p^{*} \omega^{2}
$$

in which the tensors $\underset{\sim}{f}$ and $\underset{\sim}{\mathcal{F}}$ are defined as

$$
\begin{gathered}
4 \pi \rho \omega^{2} f_{m j}(\underline{r})=-\beta_{\phi \delta_{m j}}^{2}+\psi_{m j}-\phi,_{m j} \\
4 \pi \rho \omega^{2} F_{m i j}(\underline{r})=-\left(\lambda \alpha^{2} \psi \cdot m^{\delta_{i j}}+2 \mu \beta^{2} \phi, j \delta_{m j}-2 \mu \psi \cdot m j\right.
\end{gathered}
$$

The $\psi$ - and, by implication, the $\phi$-integrals given in equations (14) and (15), etc., are the volume integrals associated with the inhomogeneous Helmholtz equation. They can be carried out for an ellipsoidal region by expanding (exp $i k R) / R$ in Taylor series expansions with respect to $r^{\prime}$ for $r>r^{\prime}$ and with respect to $r$ for $r<r^{\prime}$. Here $k$ can be either $\alpha$ or $\beta$. Detalls are given in reference 6 . This type of expansion for the integrand is particularly useful in determining the coefficients of a "polynomial" distribution of $\pi_{j}^{*}$ and $\varepsilon_{i}^{*}(1)$.

The fourth-rank tensor $S_{j k p q}$ is the connecting tensor between the eigenstrains and the applied strains, that is,

$$
\left.\begin{array}{c}
\varepsilon_{j k}^{*(1)}=S_{j k p q} \sigma_{p q}^{(a)} \\
S_{j k p q}=S_{j k p q}\left(C, a_{i}, \omega\right)
\end{array}\right\}
$$

for the case of uniform eigenstrains and eigenforces. In developing these expressions, the volume average of the $\phi$-integrals must be evaluated. Finally, note that $\rho^{*}$ and $\mathcal{C}^{*}$ are complex, where the real and imaginary parts are associated with the velocity and attenuation, respectively.

\section{Example: Spherical Inclusion Materials}

Let the spherical inclusion materials of radius a be randomly distributed over the whole volume of the matrix. If the matrix and the inhomogeneities are isotropic, the effective medium is also isotropic. It is easy to show that

$$
D_{m j}=\delta_{m j}\left\{\frac{-\left\langle f_{33}(r)\right\rangle}{f_{33}[0]+4 \pi\left(\rho^{\prime}-\rho^{*}\right) \omega^{2}} \mid=\delta_{m j} D\right.
$$

and

$$
\left.\begin{array}{l}
s_{j k p q}=s_{k j p q}=s_{j k q p}=s_{p q j k} \\
s_{1111}=s_{2222}=s_{3333}=c_{1} \\
s_{2323}=s_{1313}=s_{1212}=c_{3} \\
s_{1122}=s_{1133}=s_{2233}=c_{2}
\end{array}\right\}
$$


where

$$
\begin{gathered}
C_{1}=\left(C_{1}^{*}+C_{2}^{*}-2 G C_{2}^{*}\right) /\left[\left(C_{1}^{*}\right)^{2}+C_{1}^{*} C_{2}^{*}-2\left(C_{2}^{*}\right)^{2}\right] \\
C_{2}=\left(C_{1}^{*} G-C_{2}^{*}\right) /\left[\left(C_{1}^{*}\right)^{2}+C_{1}^{*} C_{2}^{*}-2\left(C_{2}^{*}\right)^{2}\right] \\
C_{3}=\left\{2 F_{122,1}[0]+\mu^{*} /\left(\mu^{\prime}-\mu^{*}\right)\right\} \\
C_{2}^{*}=G_{111,1}[0]+(G+1) F_{122,1}[0]+H \\
C_{1}^{*}=F_{111,1}[0]+2 G F_{122,1}[0]-F \\
F \equiv-\left(\lambda^{*}+2 \mu^{*}\right) / G \\
G=\left(\lambda^{\prime}-\lambda^{*}\right) /\left[\left(\lambda^{\prime}-\lambda^{*}\right)+2\left(\mu^{\prime}-\mu^{*}\right)\right] \\
H=\lambda^{*} / G
\end{gathered}
$$

Following the theory developed in the previous sections, the effective moduli and mass density are found to be

$$
\begin{gathered}
\rho^{*}=\rho+f \Delta \rho D \\
\lambda^{*}=\lambda+f\left[\Delta \lambda\left(A_{1111}+2 A_{1122}\right)+2 \Delta \mu A_{1122}\right] \\
\mu^{*}=\mu+f \Delta \mu\left(A_{1212}+A_{1221}\right) \\
K^{*}=K+f\left[\left(A_{1111}+2 A_{1122}\right) \Delta \lambda+(2 / 3)\left(3 A_{1122}+A_{1212}+A_{1221}\right) \Delta \mu\right]
\end{gathered}
$$

Clearly, the velocities are dispersive. At frequencies above the Rayleigh limit, this phenomenon is pronounced. From figures 10 to 12 , the bulk moduli, shear moduli, and longitudinal velocities are shown as functions of the volume concentration of spherical inclusion materials for aluminum spheres in germanium for different dimensionless wave numbers aa. For a given fixed concentration, the moduli $K^{*}$ and $\mu^{*}$ and the velocities $v_{L}$ and $v_{T}$ are increased as the dimensionless wave number a is increased. The dispersiveness of effective shear modulus is minimal and that of effective bulk modulus is more pronounced (figs. 12 and 13).

As an example of detecting localized damage by void nucleation, let all small voids be localiy nucleated within a localized small region $\Omega$ of radius $R$ (fig. 14). The effective moduli of this composite can therefore be obtained from equations (64) and (65). If void nucleation outside the region $\Omega$ can be ignored, the scattering of the composite sphere can easily be obtained. By using the computer program developed in reference 17 for that of a single spherical inclusion. the scattering cross section for a composite sphere consisting of small voids in titantum is displayed as a function of dimensionless wave number for different concentrations of voids (fig. 15). As the volume fraction of voids inside $\Omega$ is changed, the effective properties $\rho^{*}, \lambda^{*}$, and $\mu^{*}$ are also changed. Hence, the attenuation effect is pronounced as the concentration of voids is increased. The 
scattering cross section, which is essentially proportional to the attenuation (ref. 1) increases with increasing concentration $f_{r}$. It appears that these curves can be used to locate and calibrate porosity in a structural component.

\section{CONCLUDING REMARKS}

The velocity and attenuation of ultrasonic waves in two-phase media were studied by using a self-consistent averaging scheme. The effective medium had to possess the same strain and kinetic energy as the physical medium. The concept of volume averaging for physical quantities was employed. The solution depended on the scattering of a single inhomogeneity. The theory is general and can be applied to any multicomponent material system. Since the scattering of an ellipsoidal inhomogeneity is known, the average theorem presented in this report can be used to study the velocity and attenuation of distributed inhomogeneities of shapes such as disks and short fibers. The orientation of these inhomogeneities as well as their sizes, as in the spherical geometry, will necessarily induce anisotropy in the effective medium. The scheme developed herein was not compared with test data. The use of a self-consistent scheme in determining static material properties for composites has been explored (ref. 13).

Results for randomly distributed spherical inclusions of radius a are presented. Effective moduli and mass density were found to be dispersive. A simple model of localized damage was studied. It is well known that porosity is directly related to the strength of rocks and ceramics. Therefore the theoretical study of velocity and attenuation in two-phase media may be a viable means for data analysis in ultrasonic evaluation of dynamic material properties for composite bodies (ref. 14) and polycrystals (ref. 15). As in determining the static properties of materials, the problem may be more in the mechanics than in the manufacturing (ref. 16). Deformation processes such as rolling, sheet metal forming, and drawing often involve large plastic flow, moisture absorption, and thermal cycling. Residual stresses and anisotropy are introduced into the material and 1 imit the amount of deformation to fracture with a directional dependence. Theoretical development in acoustoelasticity appears to be lacking (ref. 18).

\section{REFERENCES}

1. R. True 11, C. Elbaum, and B.B. Chick, Ultrasonic Methods in Solid State Physics, Academic Press, N.Y., 1969.

2. Elastic Waves and Non-Destructive Testing of Materials, edited by Y.H. Pao, AMD-29, American Society of Mechanical Engineers, New York, 1978.

3. L.S. Fu, "Mechanics Aspects of NDE by Sound and UTtrasound," Applied Mechanics Review, vol. 35, no. 8, 1982, pp. 1047-1057.

4. L.S. Fu, "A New Micro-Mechanical Theory for Randomly Inhomogeneous Media," Symposium on Wave Propagation in Inhomogeneous Media and UTtrasonic Nondestructive Evaluation, AMD-62, June 1984, pp. 155-174.

5. L.S. Fu and T. Mura, "The Determination of Elastodynamic Fields of the Ellipsoidal Inhomogeneity," Trans. ASME J. App 1. Mech., vol. 50, 1983, pp. 390-397. 
6. L.S. Fu and T. Mura, "Volume Integrals of Ellipsoids Associated with the Inhomogeneous Helmholtz Equations," Wave Motion, vol. 4, 1982, pp. 141-149.

7. R. Hill, "The Elastic Behavior of a Crystalline Aggregate," Proc. Phys. Soc. A65, 1952, p. 319 .

8. B. Budiansky and T.T. Wu, "Theoretical Prediction of Plastic Strains of Polycrystals," Proc. 4th U.S. Nat. Cong. App1. Mech., 1962, pp. 1175-1185.

9. C.W. Bert, "Models for Fibrous Composites with Different Properties in Tension and Compression," J. Eng. Mater. Technol. ASME, vo1. 99, 1977, p. 344.

10. A.B. Schultz and S.W. Tsai, "Dynamic Moduli and Damping Ratio in FiberReinforced Composites," J. Comp. Materials, vol. 2, no. 3, 1968, pp. 368-379.

11. J. Achenbach, Wave Propagation in Elastic Solids, North Holland Pub. Co., 1975.

12. T.C.T. Ting, "Dynamic Response of Composites," Applied Mechanics Reviews, vol. 33, no. 12, Dec. 1980.

13. L.S. Fu, "On the Feasibility of Quantitative Ultrasonic Determination of Fracture Toughness - A Literature Review," International Advances in Nondestructive Testing, vol. 7, May 1981. (Also NASA CR-3356, 1980.)

14. L.S. Fu, "Scatter of Elastic Waves Due to a Thin Flat Elliptical Inhomogeneity," NASA CR-3705, 1983.

15. A. Vary, "Correlations Between U1trasonic and Fracture Toughness Factors in Metallic Materials," ASTM STP 677, pp. 563-578, 1979.

16. 2. Hashin, "Analysis of Composite Materials - A Survey," J. Appl. Mech., Trans ASME, vol. 50, 1983, p. 481 .

17. Y.C. Sheu and L.S. Fu, "The Transmission or Scattering of Elastic Waves by an inhomogeneity of Simple Geometry: A Comparison of Theories," NASA Contractor Report CR-3659, Jan. 1983.

18. H. Fukuoka, et al., "Acoustoelastic Stress Analysis of Residual Stress in the Rim of Railroad Wheels," ASME AMD-62, ed. G. Johnson, June 1983. 


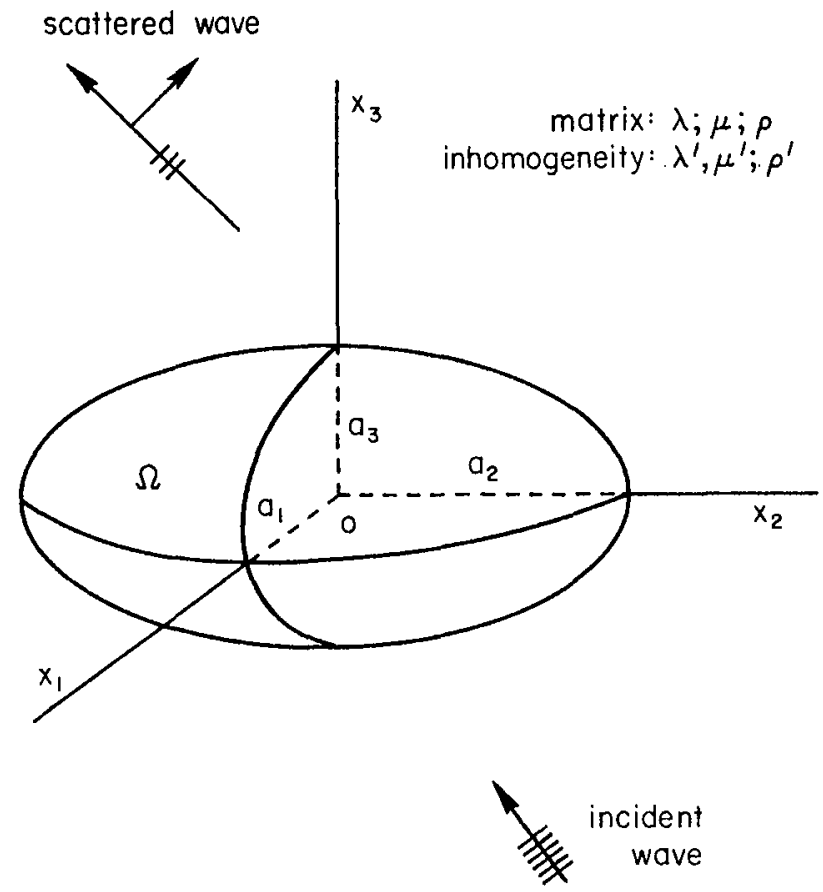

Figure 1. - Ellipsoidal inhomogeneity embedded in elastic matrix.

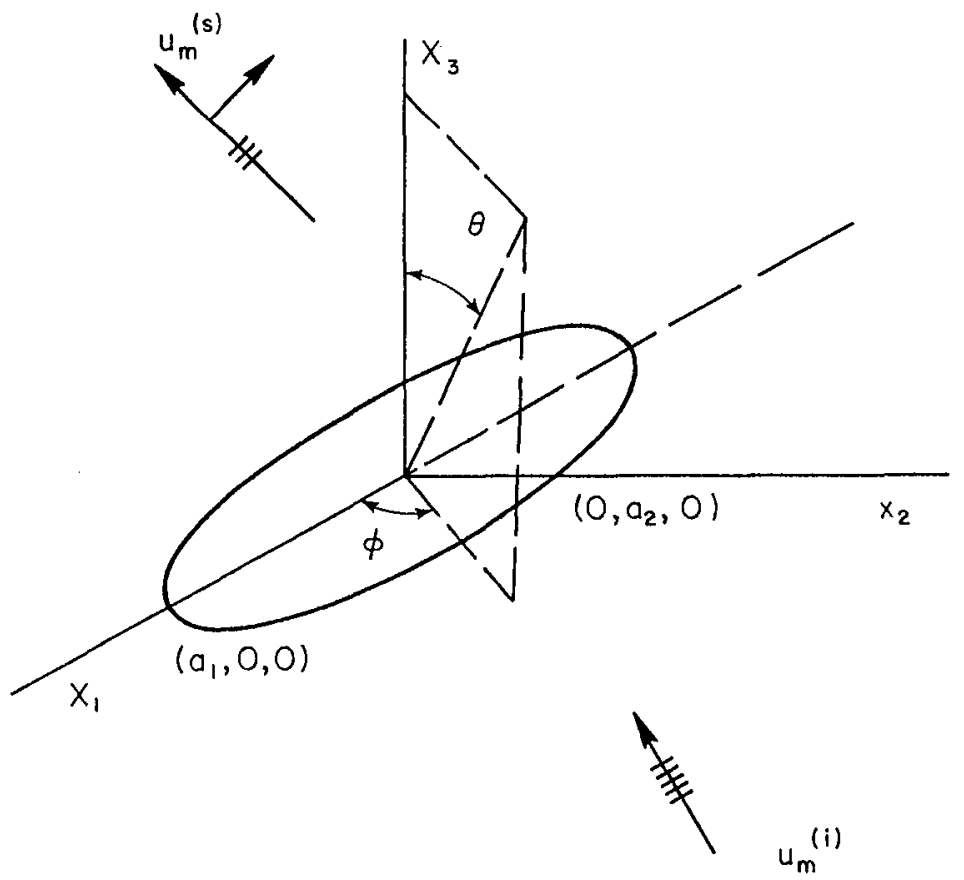

Figure 2. - Elliptical crack: $a_{3} \rightarrow 0$. 


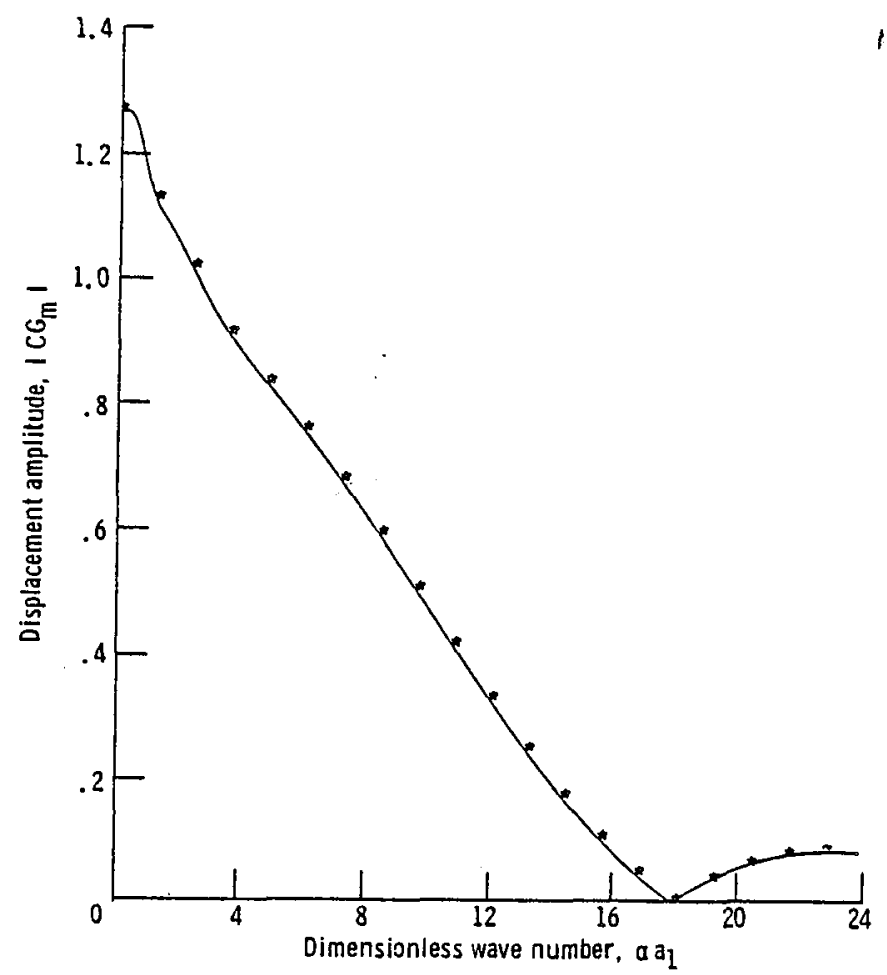

Figure 3. - $\left|C G_{m}\right|$ versus $\alpha a_{1}$ for back-scattered data for tungsten disk in aluminum matrix. $a_{2} / a_{1}=1 ; a_{1}=0.5 ; \phi=25^{\circ} ; \theta=30^{\circ} ; a_{m}=-l_{m}$.

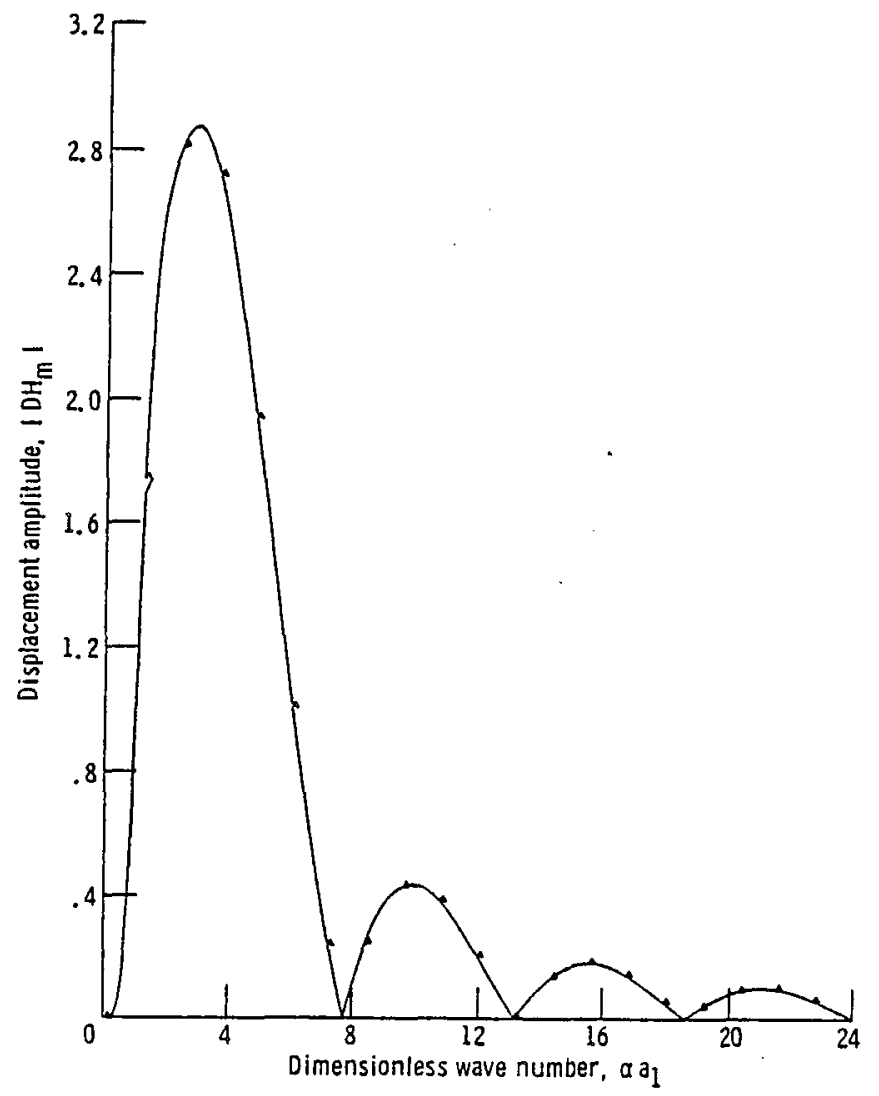

Figure 4. - $\left|\mathrm{OH}_{m}\right|$ versus $\alpha_{a}$ for back-scattered data for tungsten disk in aluminum matrix. $a_{2} / a_{1}=1 ; a_{1}=0.5 ; \phi=25^{\circ} ; \theta=30^{\circ} ; a_{m}=-l_{m}$. 


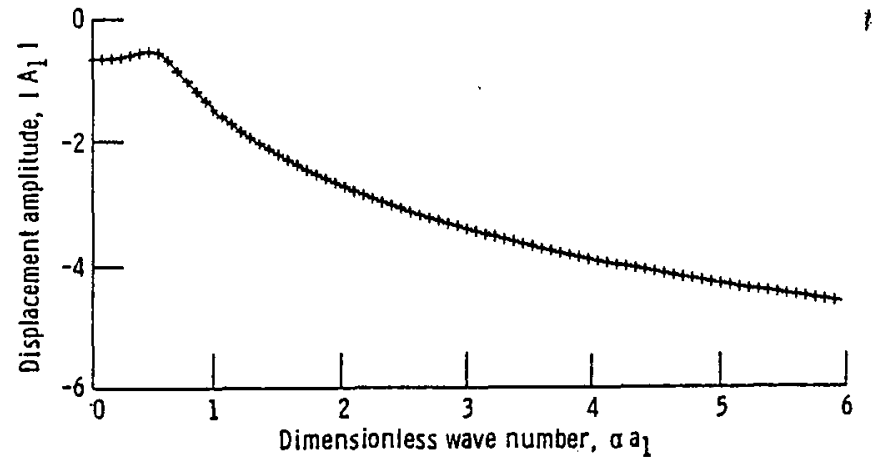

Figure 5. - $\left|A_{1}\right|$ versus $\alpha a_{1}$ for back-scattered data for tungsten disk in aluminum matrix. $a_{2} / a_{1}=1 ; a_{1}=1.0 ; \phi=25^{\circ} ; \theta=30^{\circ} ; q_{m}=-l_{m}$.

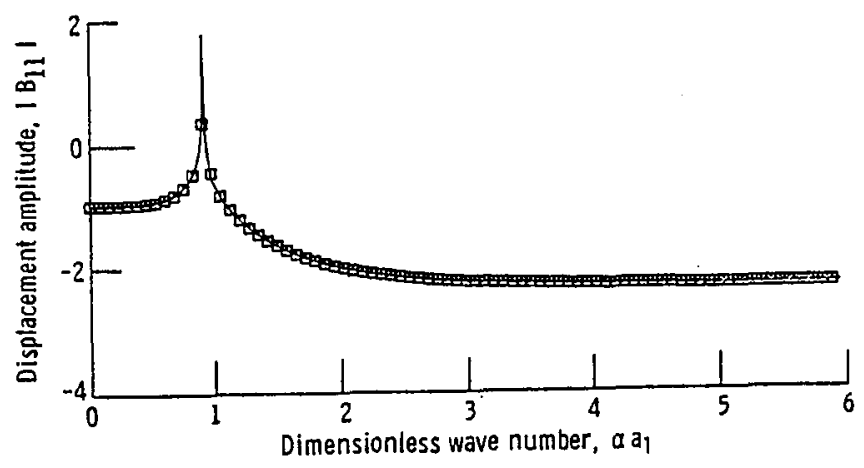

Figure 6. - $\left|B_{11}\right|$ versus $\alpha a_{1}$ for back-scattered data for tungsten disk in aluminum matrix. $a_{2} / a_{1}=1 ; a_{1}=1.0 ; \phi=25^{\circ} ; \theta=30^{\circ} ; a_{m}=-l_{m}$.

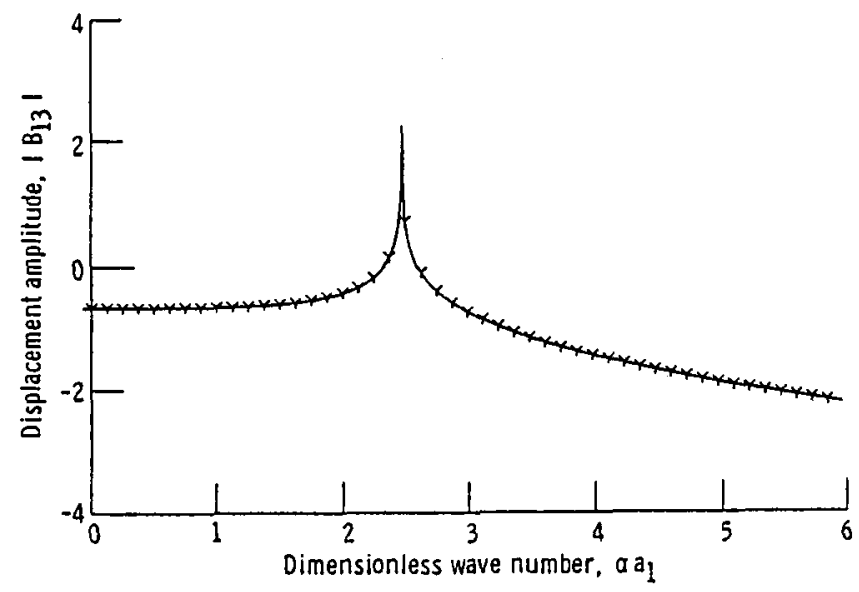

Figure 7. - $\left|B_{13}\right|$ versus $\alpha_{1} a_{1}$ for back-scattered data for tungsten disk in aluminum matrix. $a_{2} / a_{1}=1 ; a_{1}=1.0 ; \phi=25^{\circ} ; \theta=30^{\circ} ; a_{m}=-l_{m}$. 


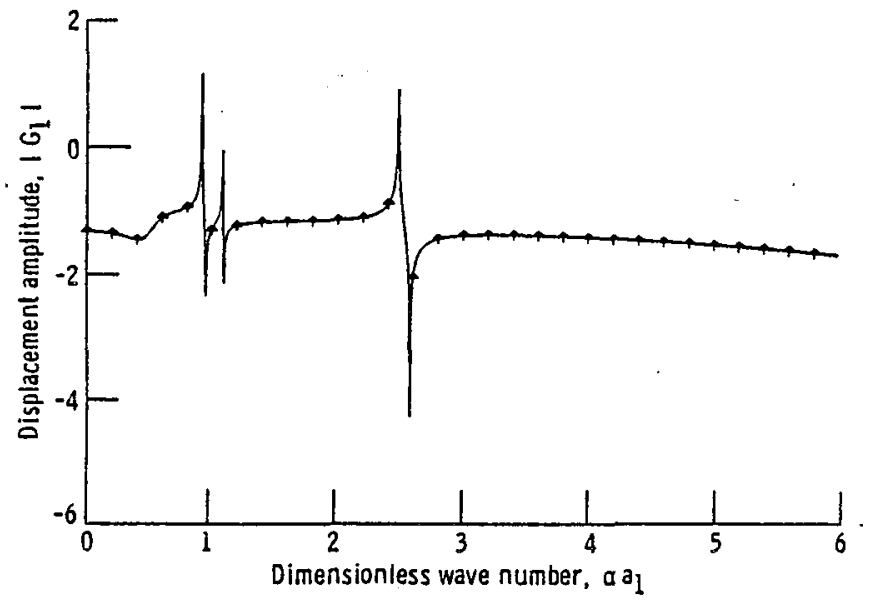

Figure 8. - $\left|G_{1}\right|$ versus $\alpha a_{1}$ for back-scattered data for tungsten disk in aluminum matrix. $a_{2} / a_{1}=1 ; a_{1}=1.0 ; \phi=25^{\circ} ; \theta=30^{\circ} ; a_{m}=-l_{m}$.

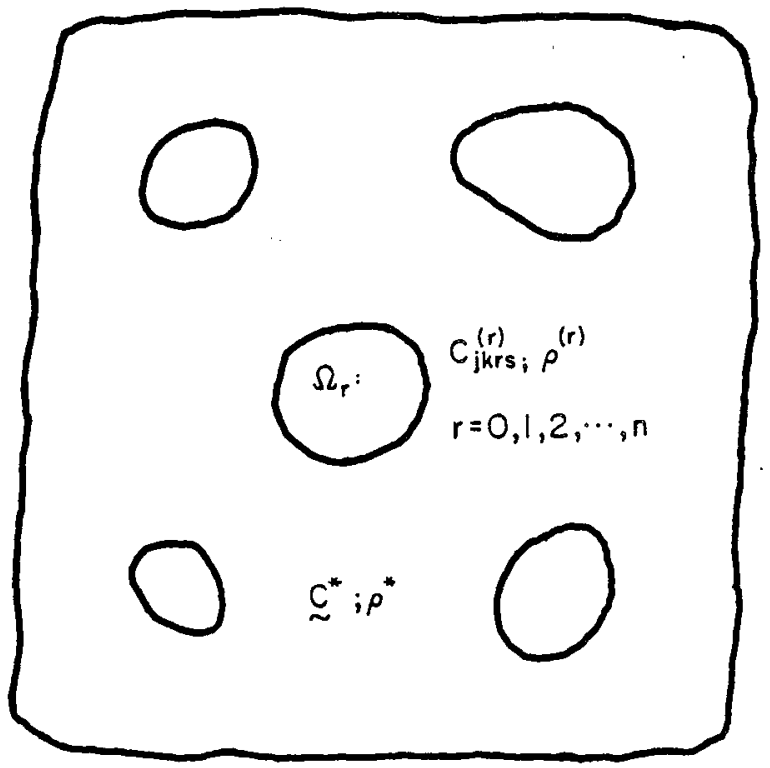

Figure 9. - Inhomogeneous medium with multicomponents. 


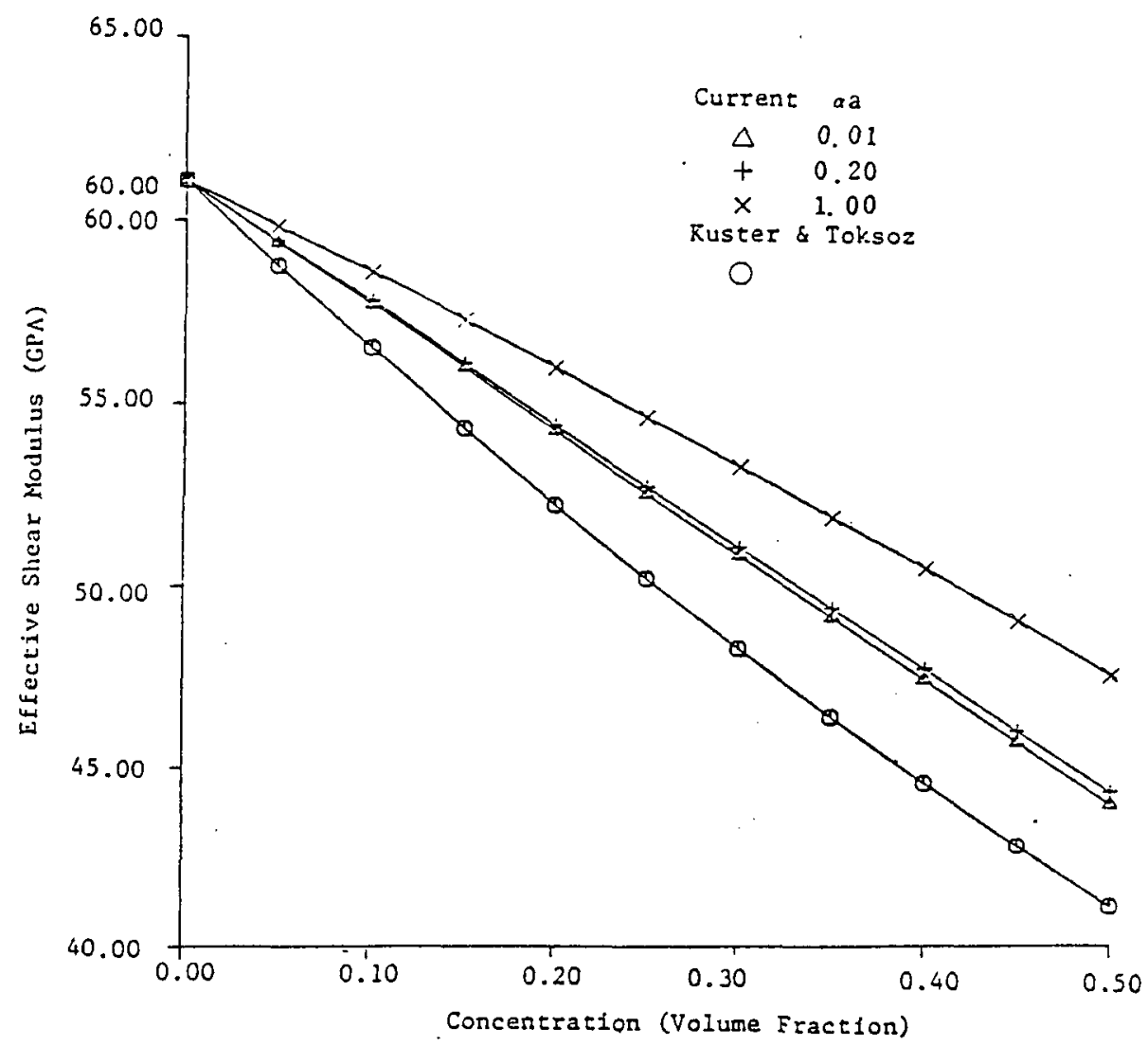

Figure 10. - Effective shear modulus versus concentration: aluminum spheres in germanium. 


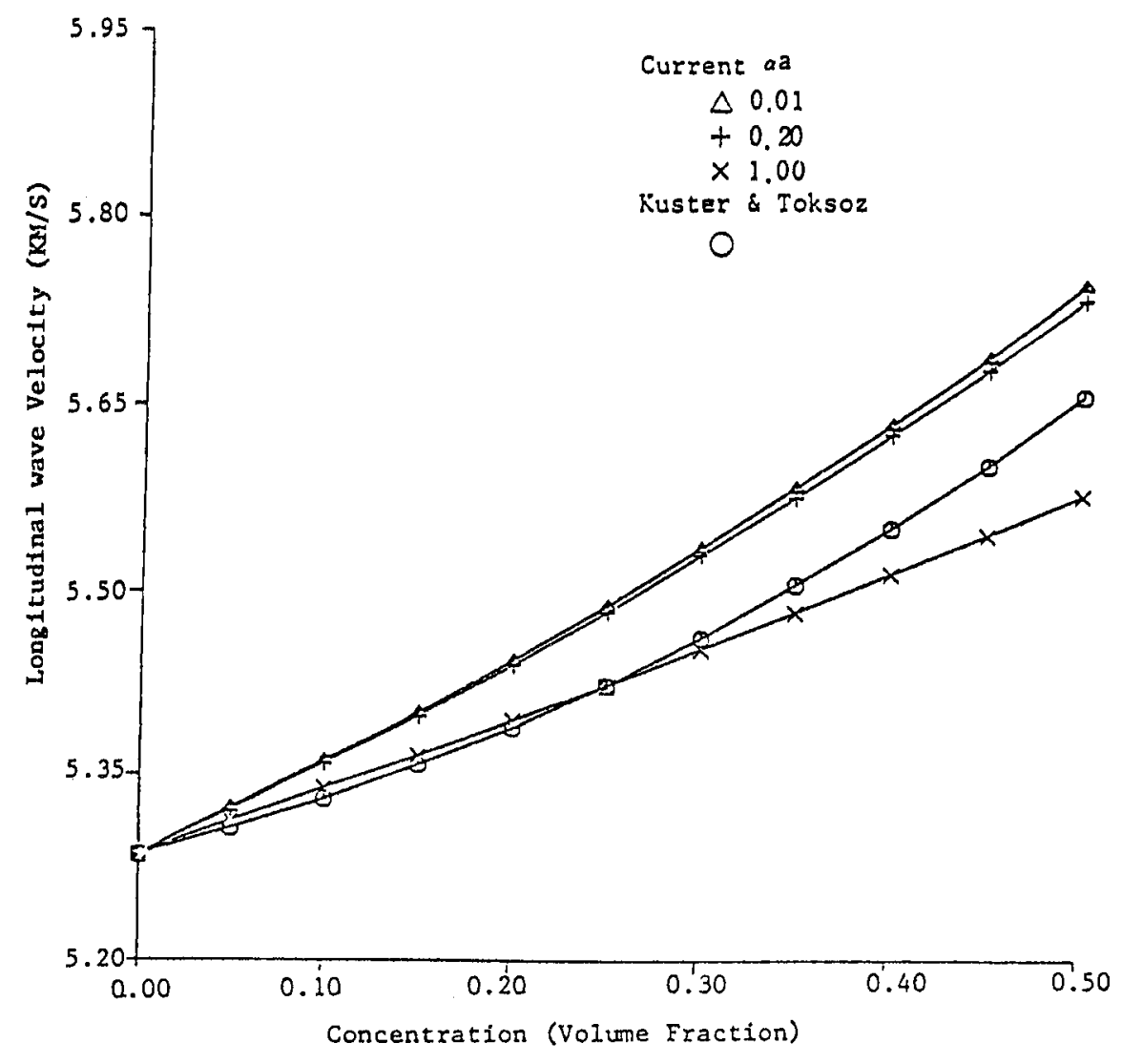

Figure 11. - Longitudinal wave velocity versus concentration: aluminum spheres in germanium.

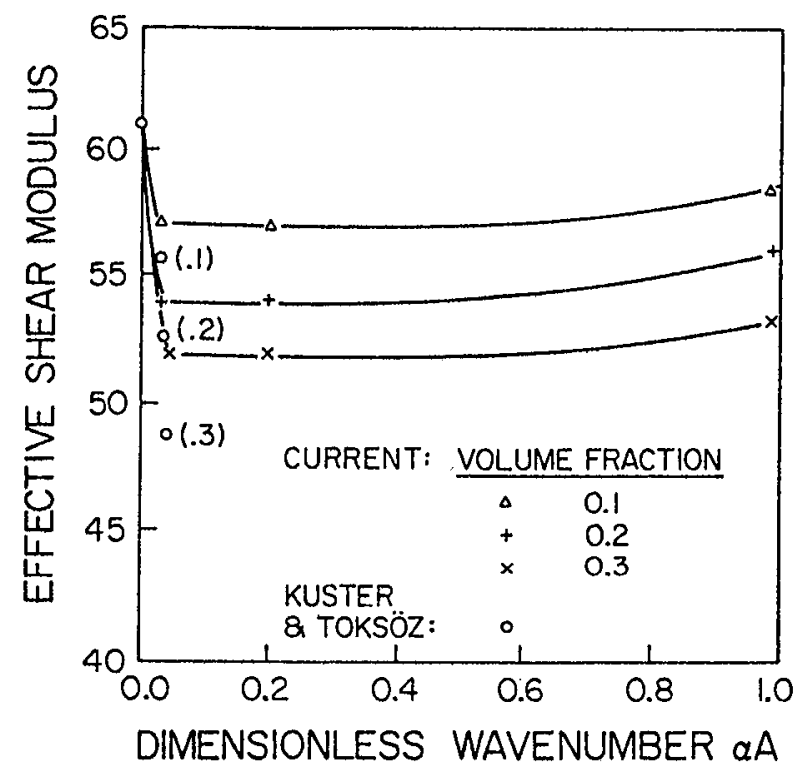

Figure 12. - Effective shear modulus versus wave number: aluminum spheres in germanium. 


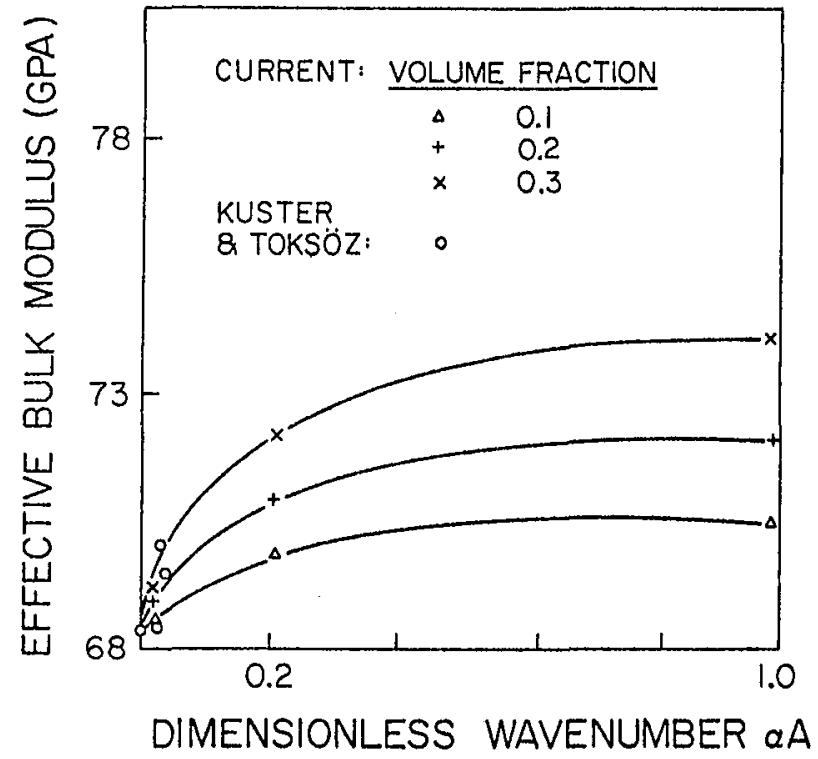

Figure 13. - Effective bulk modulus versus wave number: aluminum spheres in germanium. 


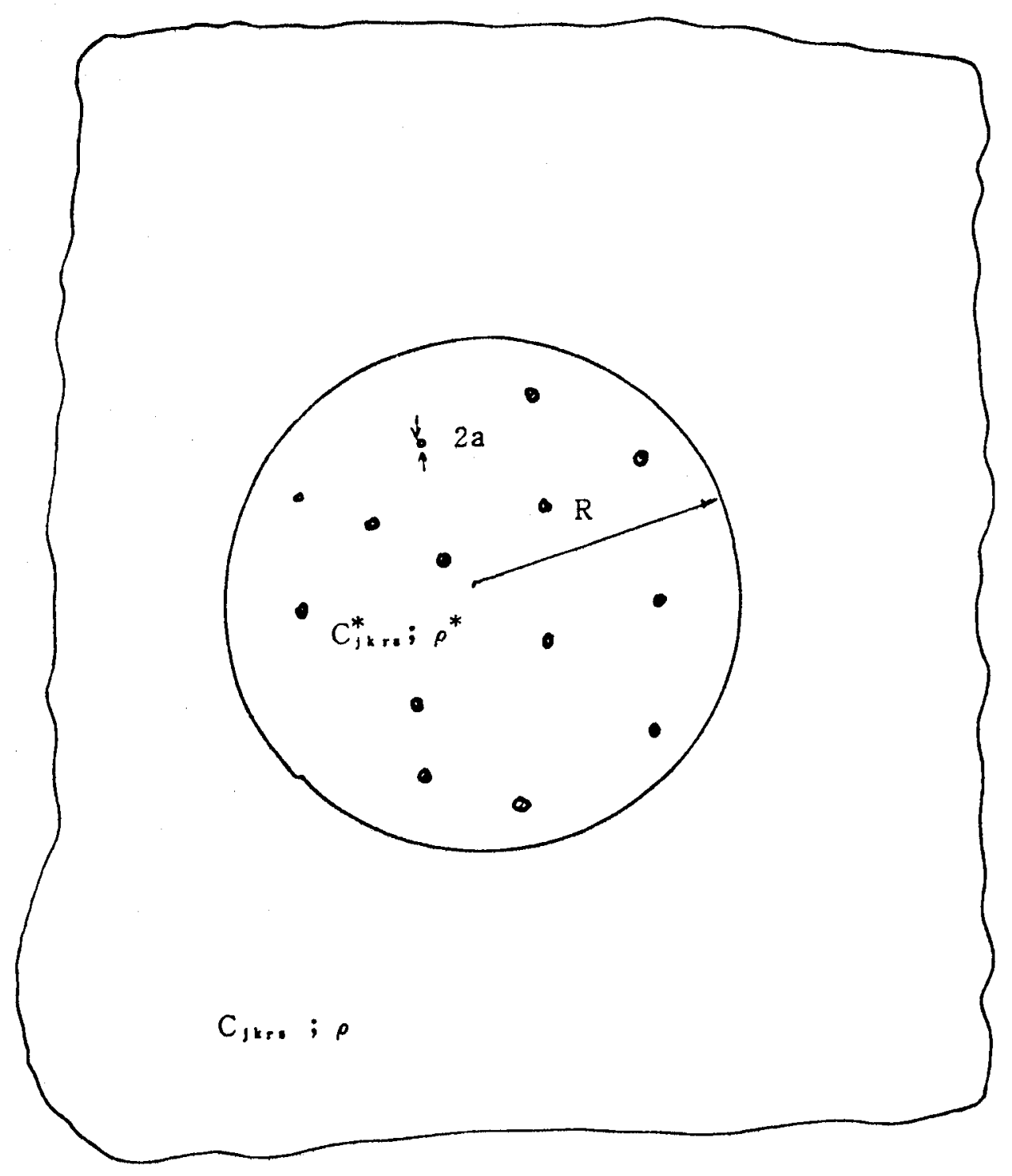

Figure 14. - Schematic diagram of localized damage in infinite solid. 


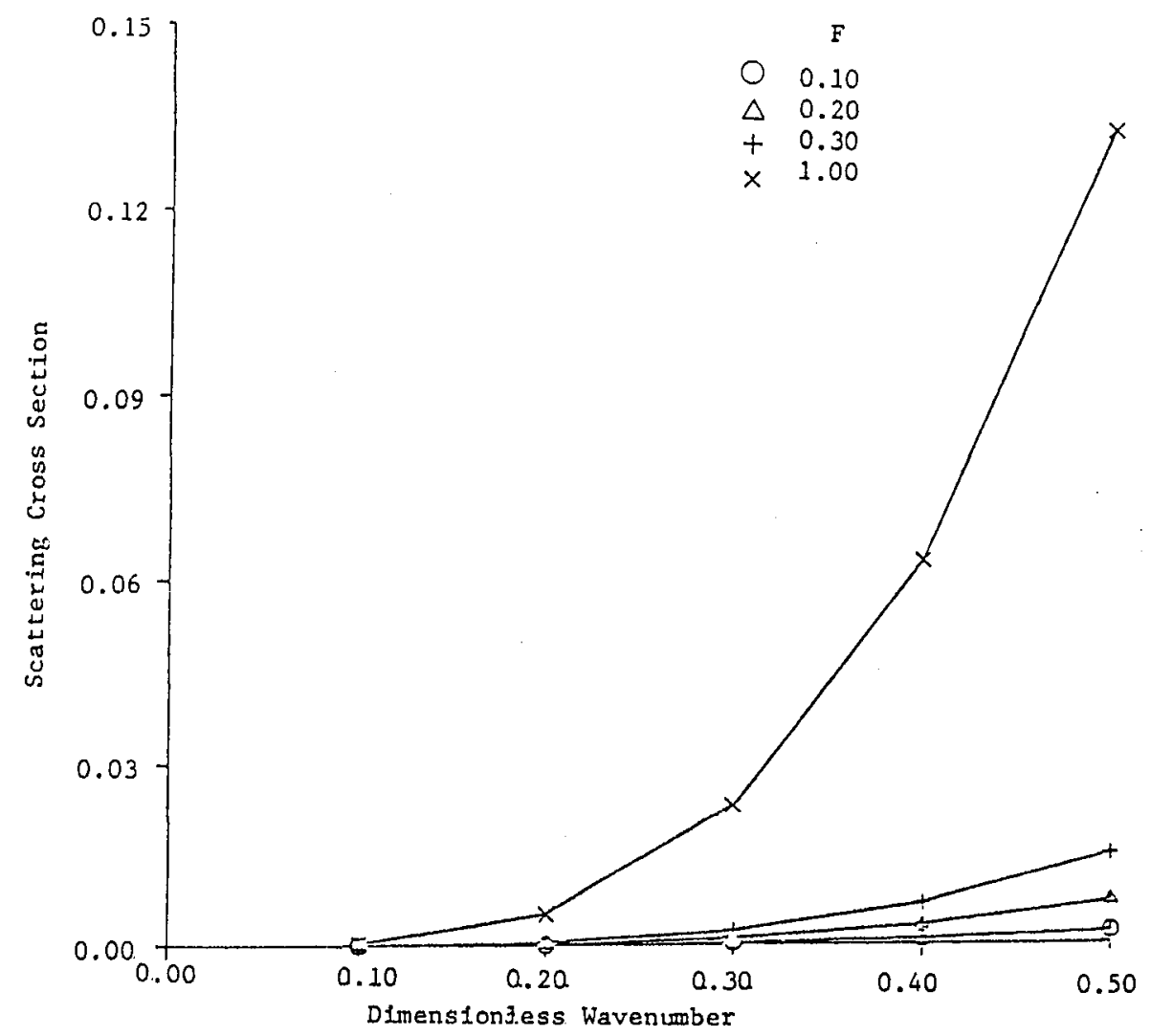

Figure 15. - Scattering cross section versus dimensionless wave number: distributed voids within a spherical domain in titanium matrix, $a / R=0.01$. 